\title{
Cytotoxic Activities of Aspergillin PZ and Trichoderone B from an Isolate of Aspergillus flavipes sp. Against NCI-60 Human Tumor Cell Lines
}

\author{
Grant C. Currens $\oplus^{1,2}$, Umesh T. Sankpal $\oplus^{2,3}$, Riyaz Basha $\oplus^{2,3}$ \\ and Eric Y. Cheng ${ }^{*}{ }^{*}$ \\ ${ }^{I}$ College of Pharmacy, University of North Texas Health Science Center, 3500 Camp Bowie \\ Boulevard, Fort Worth, TX 76107, USA \\ ${ }^{2}$ Graduate School of Biomedical Sciences, University of North Texas Health Science Center, 3500 \\ Camp Bowie Boulevard, Fort Worth, TX 76107, USA \\ ${ }^{3}$ Texas College of Osteopathic Medicine, University of North Texas Health Science Center, 3500 \\ Camp Bowie Boulevard, Fort Worth, TX 76107, USA
}

(Received March 12 2021; Revised May 25, 2021; Accepted May 26, 2021)

\begin{abstract}
In the process of piloting a new approach to natural product discovery from microorganisms isolated from systematically determined geographic sites in the State of Texas, USA, two previously identified and structurally similar bioactive pentacyclic aspochalasins, aspergillin PZ (1) and trichoderone B (2), were rediscovered from a single isolate of Aspergillus flavipes sp.. While the antimicrobial activity and cytotoxic activity of $\mathbf{1}$ had been documented in several publications, nothing was known about the bioactivities of 2 . After a large laboratory-scale fermentation and activity-guided purification, both $\mathbf{1}$ and $\mathbf{2}$ were enrolled in the NCI-60 Human Tumor Cell Lines Screen program. A preliminary single-dose assay of two natural products shows a similar cytotoxic profile and a low potency against human tumor cell lines with few exceptions at the $10 \mu \mathrm{M}$ dosage level.
\end{abstract}

Keywords: aspergillin PZ; trichoderone B; Aspergillus flavipes; natural product; cytotoxic activity (C) 2021 ACG Publications. All rights reserved.

\section{Microorganism Source}

A fungal isolate 20160726-03F1 was isolated from a dry grassy soil sample collected in July 2016 from the Concho County in central Texas, USA, and grown on soil extract agar. Fungal species identification was carried out through DNA fingerprinting of the ITS1-28S rDNA region [1]. For this, touchdown PCR was performed in a $200-\mu \mathrm{L}$ reaction tube containing one Illustra puRe-Taq PCR bead with ITS1-LR3 or LROR-LR6 primer pairs. Size of DNA amplicons was assessed through gel electrophoresis on $1 \%$ agarose gel (Figure S1) prior to purification and quantification of DNA.

\footnotetext{
* Corresponding author: E-mail: eric.cheng@unthsc.edu (E. Y. Cheng), Phone +001-817-735-0165
} 
Sanger sequencing of DNA amplicons was performed with ITS1, LROR, LR3, LR3R, or LR6 primers. An ITS1-28S contig (Figure S2; GenBank accession no. MW522866) was assembled and cleaned using GeneStudio Pro Software and ran through an NCBI-BLASTn search [2]. A. flavipes sp. was tentatively identified based on a $99.82 \%$ identity of its ITS1-28S rDNA sequence to that of $A$. flavipes NRRL 302 (Figure S3) and an inferred evolutionary history tree (Figure S4).

\section{Previous Studies}

Cytochalasans are prolific inhibitors of actin polymerization [3]. Aspochalasins, a small subgroup of cytochalasans, are a class of secondary metabolites commonly produced by many Aspergillus species and are recognizable by an isoindole unit fused to one macrocyclic ring and a C-3 2-methylpropyl group [4,5]. A. flavipes produces many cytotoxic aspochalasins including TMC-169 and aspochalasins C, D, E, I, J, and K [4,6]. These compounds have exhibited cytotoxic activity against NCI-H460 cells ( $\mathrm{IC}_{50}$ : in a range of 3.4-55.2 $\mu \mathrm{M}$ ), MCF-7 cells ( $\mathrm{IC}_{50}$ : in a range of 5.3-33.4 $\left.\mu \mathrm{M}\right)$, SF268 cells $\left(\mathrm{IC}_{50}\right.$ : in a range of 3.9-52.6 $\left.\mu \mathrm{M}\right)$ [4], and HeLa human cervical adenocarcinoma cells $\left(\mathrm{IC}_{50}\right.$ : in a range of 5.72-27.4 $\mu \mathrm{M}$ ) [7]. Aspochalasin L has demonstrated anti-HIV integrase activity ( $\mathrm{IC}_{50}$ : $71.7 \mu \mathrm{M})[8]$.

Two members of the aspochalasin family, aspergillin PZ (1) and trichoderone B (2) (Figure 1), first isolated from A. awamori [9] and Trichoderma gamsii [7], respectively, were re-discovered during our pilot exploration of a new approach to natural product discovery from microorganisms isolated from systematically determined geographic sites in the State of Texas, USA. 1 and $\mathbf{2}$ have structural similarity to aspochalasins C and D [9] with the notable difference of an internal epoxy group on the macrocycle and are well documented in their biomimetic synthesis [10].

1 was previously shown to have antimicrobial activity against Staphylococcus epidermidis [11], Klebsiella pneumonia and Pseudomonas aeruginosa [12]. Furthermore, micromolar potency of naturally and synthetically derived 1 was observed when screened against HL-60 cells (IC 50 : 56.61 $\mu \mathrm{M}), \mathrm{A} 2780$ human ovarian carcinoma cells $\left(\mathrm{IC}_{50}:>5 \mu \mathrm{M}\right)$, PC3 human prostate adenocarcinoma cells $\left(\mathrm{IC}_{50}:>25 \mu \mathrm{M}\right)$, and $\mathrm{LNCaP}$ androgen-sensitive human prostate adenocarcinoma cells $\left(\mathrm{IC}_{50}:>50 \mu \mathrm{M}\right)$ $[13,14]$.

2 differs from 1 by a C-7 hydroxy group addition on the isoindole ring [7] and until now had not been reported to be produced by A. flavipes; $\mathbf{2}$ has been studied much less extensively with no documented cytotoxicity. This report describes the comparative cytotoxic activities of $\mathbf{1}$ and $\mathbf{2}$ from a preliminary single-dose $(10 \mu \mathrm{M})$ assay of two natural products enrolled in the NCI-60 Human Tumor Cell Lines Screen program.

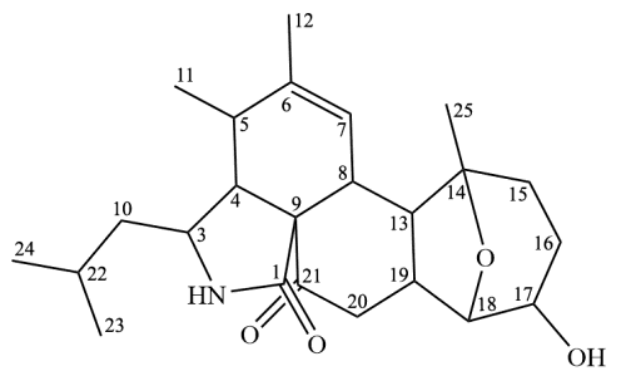

1

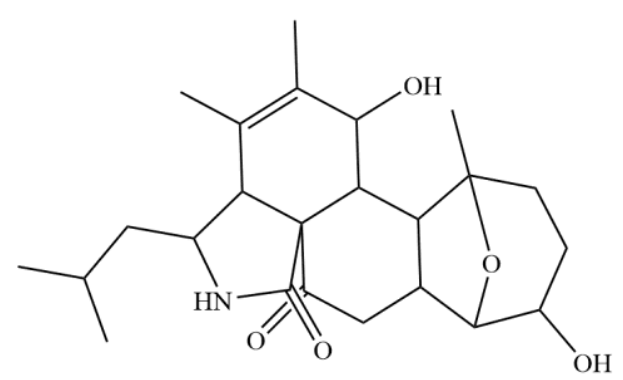

2

Figure 1. Planar structures of aspergillin PZ (1) and trichoderone B (2), which only differ by a C-7 hydroxyl group 
Cytotoxic activities of aspergillin $\mathrm{PZ}$ and trichoderone $\mathrm{B}$

\section{Present Study}

\subsection{General Experimental Procedures}

For DNA amplification, PCR was performed with Illustra puReTaq Ready-To-Go PCR beads (Cytiva Life Sciences, Marlborough, MA, USA). DNA concentrations and purity were measured with a Take $2 \mu \mathrm{L}$ micro-volume plate on a Synergy H1 plate reader with Gen5 software (BioTek, Winooski, VT, USA). Evolutionary analyses of the ITS1-28S rDNA region were conducted in MEGA $\mathrm{X}$ [15]. Pre-fractionation was performed on an AI-580S flash chromatography system (Yamazen Science, Burlingame, CA, USA) equipped with a $230 \mathrm{~nm}$ UV detector (UVD) and tandem evaporative light scattering detector (ELSD). Elution was carried out on an ODS C18 filled separation column. Semi-preparative and analytical HPLC were performed on an HPLC system equipped with a PrepStar 218 solvent delivery system (Agilent, Santa Clara, CA, USA) and Infinity 1260 diode array detector measuring at $210 \mathrm{~nm}$ and $230 \mathrm{~nm}$. HPLC separation occurred on a Zorbax StableBond-Phenyl semipreparative column $(5 \mu \mathrm{m}, 9.4 \times 250 \mathrm{~mm}$, Agilent) and analytical HPLC was carried out on a Zorbax StableBond-Aq column $(5 \mu \mathrm{m}, 4.6 \times 250 \mathrm{~mm}$, Agilent). Purification was guided by cell viability testing against human pancreatic carcinoma MIA PaCa-2 cells (ATCC ${ }^{\circ}$ CRL-1420 ${ }^{\mathrm{TM}}$; not presented) measured by the CellTiter-Glo ${ }^{\circledR}$ luciferase luminescence assay (Promega, Madison, WI, USA) 48 hrs after addition of $20 \mathrm{mg} / \mathrm{ml}$ semi-purified extract to 2,000 cells/well in triplicate [16] and analyzed on the same Synergy H1 plate reader with Gen5 software. ${ }^{1} \mathrm{H}-\mathrm{NMR}(300 \mathrm{MHz})$ on a Fourier $300 \mathrm{HD}$ (Bruker, Billerica, MA, USA), Time-of-flight (TOF) ESI-MS was performed on an Agilent 6230 TOF LC/MS. Determination of 2 was aided by comparative MS(n) fragmentation on a LTQ Orbitrap XL Ion Trap Mass Spectrometer (ThermoFisher Scientific, Waltham, MA, USA). The evolutionary history of 20160726-03F1 was inferred by using the Maximum Likelihood method and Tamura-Nei model [17]. The bootstrap consensus tree (Figure S4) was inferred from 1000 replicates [18] and taken to represent the evolutionary history of the taxa analyzed [18]. Branches corresponding to partitions reproduced in less than $50 \%$ bootstrap replicates were collapsed. Initial tree(s) for the heuristic search were obtained automatically by applying Neighbor-Join and BioNJ algorithms to a matrix of pairwise distances estimated using the Tamura-Nei model, and then selecting the topology with superior log likelihood value. A discrete Gamma distribution was used to model evolutionary rate differences among sites [5 categories $(+G$, parameter $=0.2454)$ ]. This analysis involved 33 nucleotide sequences taken from the GenBank database.

\subsection{Fermentation, Extraction and Purification}

A. flavipes sp. was fermented statically in $12 \times 1 \mathrm{~L}$ Erlenmeyer flasks each containing $8 \mathrm{~g}$ of Cheerios breakfast cereal [19] and $48 \mathrm{~mL}$ of sucrose solution (30\% w/v), and supplemented with $1 \mathrm{~mL}$ of sodium butyrate solution $(25 \mathrm{mM})$ for three weeks at room temperature. Following fermentation, secondary metabolites were extracted by vigorously agitating the dried fungal mass with ethyl acetate (EtoAc) followed by methanol $(\mathrm{MeOH})$. The solvent extracts were then concentrated separately with a rotary evaporator yielding a total of $8.72 \mathrm{~g}$ of crude extract. Crude extracts were resuspended in EtoAc or $\mathrm{MeOH}$ and combined into a $50 \mathrm{~mL}$ beaker containing $18.0 \mathrm{~g}$ of silica gel and allowed to air-dry in a ventilated chemical hood until a fine powder was observed.

In multiple repeated rounds of fractionation, a small injection column $(15 \times 85 \mathrm{~mm})$ was dryloaded with approximately $1 \mathrm{~g}$ crude extract:silica gel mixture and mounted above a 2L ODS-C18 filled separation column on a flash chromatography system. The column was eluted with a 30-minute $\mathrm{MeOH}: \mathrm{H}_{2} \mathrm{O}$ gradient from $20 \%$ to $100 \% \mathrm{MeOH}$ followed by 30 minutes of isocratic $100 \% \mathrm{MeOH}$ elution at a constant flow rate of $10 \mathrm{~mL} / \mathrm{min}$. Eluate was monitored by $230 \mathrm{~nm} \mathrm{UV}$ detection in tandem with ELSD. Each distinct peak on the chromatogram was collected separately and concentrated through centrifugal evaporation followed by lyophilization.

The dried fraction containing 1 and $\mathbf{2}$ was resuspended in 30\% acetonitrile (ACN) and $200 \mu \mathrm{L}$ per-run was manually injected into an HPLC system and eluted through a Zorbax StableBond-Phenyl semi-preparative column. Elution occurred on an $\mathrm{ACN}: \mathrm{H}_{2} \mathrm{O}$ gradient from $20 \%$ to $80 \%$ ACN over 55 
minutes. 1 and $\mathbf{2}$ were collected separately and further purified by resuspending the dried compound in $30 \% \mathrm{MeOH}$ and injecting $50 \mu \mathrm{L}$ per-run into the same HPLC system with a Zorbax StableBond-Aq analytical column. Elution occurred on a $\mathrm{MeOH}: \mathrm{H}_{2} \mathrm{O}$ gradient from $30 \%$ to $70 \% \mathrm{MeOH}$ over 55 minutes. Throughout the purification process, the active compounds were tracked by activity-guided purification. For this, $20 \mathrm{mg} / \mathrm{mL}$ of each semi-purified extract was measured for cytotoxicity to MIA PaCa-2 pancreatic cancer cells as measured by CellTiter-Glo ATP-luciferase assay. Final yields of 1 and 2 with a greater than $90 \%$ purity were $11.8 \mathrm{mg}$ and $11.2 \mathrm{mg}$, respectively.

Comparison of TOF-ESI-MS (Figures S5 and S6) and ${ }^{1} \mathrm{H}-\mathrm{NMR}$ spectra (Figure S7) to historical MS, NMR and structural data [7,9] identified $\mathbf{1}$ and 2, which were then confirmed through stepwise MS(n) fractionation (Figure S8).

\subsection{Cytotoxic Activities of Aspergillin PZ (1) and Trichoderone B (2)}

Ten mg of each $\mathbf{1}$ and $\mathbf{2}$ was further purified through one more round of semi-preparative HPLC as described above to reach a greater than $96 \%$ purity. They were subsequently enrolled in the U.S. National Cancer Institute's NCI-60 Human Tumor Cell Lines Screen program (https://dtp.cancer.gov/discovery_development/nci-60/). For the screening, 5,000 to 40,000 cells/well depending on doubling time are plated into 96 well microtiter plates and incubated at $37^{\circ} \mathrm{C}$ and $5 \%$ $\mathrm{CO}_{2}$ for $24 \mathrm{~h}$. Following compound addition, the cells are incubated further for $48 \mathrm{~h}$ in the same conditions prior to growth inhibition assessment. Adherent and non-adherent cells are fixed with TCA (10\% and $16 \%$, respectively) incubated for 60 minutes at $4{ }^{\circ} \mathrm{C}$ then washed and air dried. Sulforhodamine B $(100 \mu \mathrm{l})$ in $1 \%$ acetic acid is added and incubated for 10 minutes at room temperature. Bound stain is solubilized in $10 \mathrm{mM}$ trizma base and absorbance is read at $515 \mathrm{~nm}$ [20].

NCI-60 screening is a well-established, reliable and robust procedure used for testing thousands of samples every year. The initial screening only uses a single dose of $10 \mu \mathrm{M}$ concentration and is not replicated; therefore, no statistical significance can be generated from the data of the initial screening.

At $10 \mu \mathrm{M}$ preliminary screening concentration, a greater than $25 \%$ reduction in growth was seen in UACC-257 melanoma cells ( 2 only), HOP-92 lung adenocarcinoma cells, A498 kidney carcinoma cells, and SNB-75 astrocytoma cells (Table 1). The cytotoxic activity of $\mathbf{1}$ or $\mathbf{2}$ did not appear to be very potent.

For CCRF-CEM acute lymphoblastic leukemia cells, HL-60 cells, T-47D ductal carcinoma cells and RPMI-8226 plasmacytoma cells, 1 was inactive at $10 \mu \mathrm{M}$ whereas $\mathbf{2}$ exerted a $>22 \%$ reduction of growth (Table 1), indicating a certain degree of cytotoxic activity differentiation between 1 and 2 .

The entire panel of NCI-60 human tumor cell lines screening result are presented in Figures S9 and S10. Due to their generally weak cytotoxic activities, neither $\mathbf{1}$ nor $\mathbf{2}$ advanced to the next step of activity screening at NCI.

Table 1. Percentage of cell growth* of select NCI-60 cell lines following exposure of a $10 \mu \mathrm{M}$ single dose of $\mathbf{1}$ or $\mathbf{2}$. The standard initial NCI-60 screening was not replicated; therefore, no error bars can be generated from the dataset.

\begin{tabular}{cccccccccc}
\hline Compounds & $\begin{array}{c}\text { UACC- } \\
\mathbf{2 5 7}\end{array}$ & $\begin{array}{c}\text { HOP- } \\
\mathbf{9 2}\end{array}$ & $\mathbf{A 4 9 8}$ & $\begin{array}{c}\text { SNB- } \\
\mathbf{7 5}\end{array}$ & $\begin{array}{c}\text { CCRF- } \\
\text { CEM }\end{array}$ & HL-60 & T-47D & $\begin{array}{c}\text { RPMI- } \\
\mathbf{8 2 2 6}\end{array}$ & $\begin{array}{c}\text { NCI-60 } \\
\text { Mean }\end{array}$ \\
\hline $\mathbf{1}$ & 80.92 & 71.33 & 58.49 & 48.94 & 111.65 & 99.70 & 98.00 & 108.59 & 94.31 \\
$\mathbf{2}$ & 73.26 & 65.11 & 62.06 & 48.98 & 84.70 & 75.89 & 75.49 & 86.22 & 88.33 \\
$\Delta$ & 7.66 & 6.22 & 3.57 & 0.04 & 26.95 & 23.81 & 22.51 & 22.37 & 5.98 \\
\hline
\end{tabular}

* Control group assumes a $100 \%$ cell growth.

$\Delta$ indicates the difference in cell growth percentage $\mathbf{1}$ and $\mathbf{2}$.

It is not surprising that widespread potent cytotoxic activity was not observed at the $10 \mu \mathrm{M}$ dosage level as most documented $\mathrm{IC}_{50}$ values of $\mathbf{1}$ in other cell lines have been $>25 \mu \mathrm{M}[13,14]$. It is 


\section{Cytotoxic activities of aspergillin PZ and trichoderone $\mathrm{B}$}

however worth noting a $>50 \%$ reduction of cell growth in SNB-75 cells given the relatively low potency of both compounds in other human tumor cell lines suggesting the potential for selective cell specific activity.

Our NCI-60 results and previous bioactivity comparisons of related aspochalasins [4,6] indicate that there is room for enhancing potency of $\mathbf{1}$ by assessing structure-activity relationships of the macrocyclic R groups, particularly at C-17, $-18,-19$, and -20 or C-7 of the isoindole ring (Figure 1). While the mechanisms of action for $\mathbf{1}$ and $\mathbf{2}$ are presently unknown, their presumed precursor, cytochalasin $\mathrm{D}$ [10], has been shown to block actin polymerization [3,21-23] by binding and capping the elongation site $[23,24]$. If these aspochalasins work similarly, they could presumably be interrupting the cytokinesis [23], adhesion, motility, or signaling [25] of rapidly dividing cancer cells. Determining the mechanism of action of both molecules would aid in optimizing their activity.

\section{Acknowledgments}

We thank Dr. Nigam Mishra and Alshaima'a Qunies (University of North Texas Health Science Center, UNTHSC) for their assistance in procuring and analyzing the NMR data and Dr. Lazlo Prokai (UNTHSC) for assistance in procuring the MS/MS data to aid in finalizing compound identification.

\section{Supporting Information}

Supporting Information accompanies this paper on http://www.acgpubs.org/journal/recordsof-natural-products

\section{ORCID}

Grant C. Currens: 0000-0001-9344-3684

Umesh T. Sankpal: 0000-0001-6696-2315

Riyaz Basha: 0000-0002-4071-0993

Eric Y. Cheng: 0000-0001-9336-2593

\section{References}

[1] H. A. Raja, A. N. Miller, C. J. Pearce and N. H. Oberlies (2017). Fungal identification using molecular tools: A primer for the natural products research community, J. Nat. Prod. 80 (3), 756-770

[2] Z. Zhang, S. Schwartz, L. Wagner and W. Miller (2000). A greedy algorithm for aligning DNA sequences, J. Comput. Biol. 7 (1-2), 203-214

[3] H. Ohmori, S. Toyama and S. Toyama (1992). Direct proof that the primary site of action of cytochalasin on cell motility processes is actin, J. Cell Biol. 116 (4), 933-941

[4] G. X. Zhou, E. M. Wijeratne, D. Bigelow, L. S. Pierson, H. D. VanEtten and A. A. Gunatilaka (2004). Aspochalasins I, J, and K: three new cytotoxic cytochalasans of Aspergillus flavipes from the rhizosphere of Ericameria laricifolia of the Sonoran Desert, J. Nat. Prod. 67 (3), 328-332

[5] X. Li, W. Ding, P. Wang and J. Xu (2018). Two novel aspochalasins from the gut fungus Aspergillus sp. Z4, Mar. Drug. 16 (10), 00343 (9 pages).

[6] J. Kohno, N. Nonaka, M. Nishio, T. Ohnuki, K. Kawano, T. Okuda and S. Komatsubara (1999). TMC169, a new antibiotic of the aspochalasin group produced by Aspergillus flavipes, J. Antibiot. 52 (6), 575-577

[7] G. Ding, H. Wang, L. Li, A. J. Chen, L. Chen, H. Chen, H. Zhang, X. Liu and Z. Zou (2012). Trichoderones A and B: Two pentacyclic cytochalasans from the plant endophytic fungus Trichoderma gamsii, Eur. J. Org. Chem. 2012 (13), 2516-2519

[8] S. Rochfort, J. Ford, S. Ovenden, S. S. Wan, S. George, H. Wildman, R. M. Tait, B. Meurer-Grimes, S. Cox, J. Coates and D. Rhodes (2005). A novel aspochalasin with HIV-1 integrase inhibitory activity from Aspergillus flavipes, J. Antibiot. 58 (4), 279-283

[9] Y. Zhang, T. Wang, Y. Pei, H. M. Hua and B. Feng (2002). Aspergillin PZ, a novel isoindole-alkaloid from Aspergillus awamori, J. Antibiot.. 55, 693-695 
[10] J. R. Reyes, N. Winter, L. Spessert and D. Trauner (2018). Biomimetic synthesis of (+)-Aspergillin PZ, Angew. Chem. Int. Edit. 57 (47), 15587-15591

[11] C. J. Zheng, C. L. Shao, L. Y. Wu, M. Chen, K. L. Wang, D. L. Zhao, X. P. Sun, G. Y. Chen and C. Y. Wang (2013). Bioactive phenylalanine derivatives and cytochalasins from the soft coral-derived fungus, Aspergillus elegans, Mar. Drug. 11 (6), 2054-2068

[12] Y. Erden, S. Tekin, K. Ceylan, C. Tekin and S. Kirbag (2019). Antioxidant, Antimicrobial and Anticancer Activities of the Aspergillin PZ and Terphenyllin Secondary Metabolites: An in vitro Study. Gazi Univ. J. Sci. 32, 792-800

[13] L. Chen, Y. T. Liu, B. Song, H. W. Zhang, G. Ding, X. Z. Liu, Y. C. Gu and Z. M. Zou (2014). Stereochemical determination of new cytochalasans from the plant endophytic fungus Trichoderma gamsii. Fitoterapia 96, 115-122

[14] S. M. Canham, L. E. Overman and P. S. Tanis (2011). Identification of an unexpected 2oxonia[3,3]sigmatropic rearrangement/aldol pathway in the formation of oxacyclic rings. Total synthesis of (+)-aspergillin PZ, Tetrahedron. 67 (51), 9837-9843

[15] S. Kumar, G. Stecher, M. Li, C. Knyaz and K. Tamura (2018). MEGA X: Molecular evolutionary genetics analysis across computing platforms, Mol. Biol. Evol. 35 (6), 1547-1549

[16] S. Shelake, U. T. Sankpal, D. Eslin, W. P. Bowman, J. W. Simecka, S. Raut, A. Ray and R. Basha (2019). Clotam enhances anti-proliferative effect of vincristine in Ewing sarcoma cells, Apoptosis. 24 (1-2), 21-32

[17] K. Tamura and M. Nei (1993). Estimation of the number of nucleotide substitutions in the control region of mitochondrial DNA in humans and chimpanzees, Mol. Biol. Evol. 10 (3), 512-526

[18] J. Felsenstein (1985). Confidence limits on phylogenies: An approach using the bootstrap. Evolution. 39 (4), 783-791

[19] B. Wang, J. You, J. B. King, S. Cai, E. Park, D. R. Powell and R. H. Cichewicz (2014). Polyketide glycosides from bionectria ochroleuca inhibit Candida albicans biofilm formation, J. Nat. Prod. 77 (10), 2273-2279

[20] R. H. Shoemaker (2006). The NCI60 human tumour cell line anticancer drug screen, Nat. Rev. Cancer. 6 (10), 813-823

[21] J. J. Otto (1994). Actin-bundling proteins, Curr. Opin. Cell Biol. 6 (1), 105-109

[22] E. Friederich, T. E. Kreis and D. Louvard (1993). Villin-induced growth of microvilli is reversibly inhibited by cytochalasin D, J. Cell. Sci. 105, 765-775

[23] K. Scherlach, D. Boettger-Schmidt, N. Remme and C. Hertweck (2010). The chemistry and biology of cytochalasans, Nat. Prod. Rep. 27, 869-886

[24] D. C. Lin, K. D. Tobin, M. Grumet and S. Lin (1980). Cytochalasins inhibit nuclei-induced actin polymerization by blocking filament elongation, J. Cell Biol. 84 (2), 455-460

[25] E. Skellam (2017). The biosynthesis of cytochalasans, Nat. Prod. Rep. 34 (11), 1252-1263

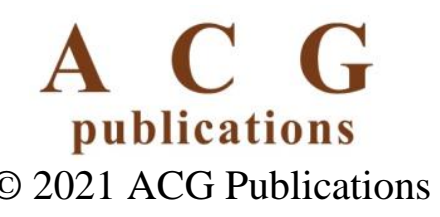

